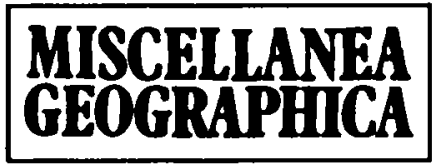

WARSZAWA 1992 Vol. 5

Elżbieta Wolk-Musial, Andrzej Musiał

\title{
L'UTILISATION DES UNITÉS PHOTOMORPHIQUES DANS L'ÉTUDE DU RELIEF DES RÉGIONS POLAIRES
}

Le relief du terrain apparaît avec le plus de netteté sur les photos aériennes grâce à l'effet stéréoscopique. Sous les latitudes moyennes, les formes du relief ne sont pas toujours directement visibles sur le matériel de télédétection, étant souvent cachées sous la végétation, dissimulées par des modes d'utilisation du sol ou parfois par la couverture du sol.

Il est beaucoup plus facile d'étudier les relations entre l'aspect des unités photomorphiques et les types génétiques du relief dans les terrains à l'environnement naturel intact. Ce type d'environnement caractérise encore les terrains polaires dont la région de Berzeliusdalen (Spitsberg occidental) au relief diversifié et découvert.

L'analyse par photointerprétation a été effectuée à partir des photos aériennes panchromatiques au 1:46 000 prises le 9 juillet 1960 par le Norsk Polarinstitut.

L'analyse stéréoscopique du matériel de télédétection a permis de distinguer, dans le terrain étudié, 23 superficies différant les unes des autres par le phototon, la structure et la texture (Fig.1, Tab.1). Les unités distinguées ont été rangées dans quatre groupes de phototons, à savoir: blanc, gris, gris clair et gris foncé constituant le fond de structures qui définissent la forme de l'élément créant l'image. On a reconnu les structures suivantes: amorphes, à bandes, rayées, tachetées. On a également analysé la composition d'éléments constituant la photo aérienne et inscrits dans la texture chaotique, parallèle, concentrique, dendritique, à grilles, à tresses, à rayons et poreuse. Parfois, les superficies sélectionnées, au phototon et à la structure similaires, ne se distinguent que par la texture comme le démontre le cas des unités 10 et 11 .

Les superficies des unités photomorphiques ont été soumises à une analyse géomorphologique effectuée à partir de l'interprétation détaillée des photos aériennes, et d'études géomorphologiques sur le terrain datant de 1988. Cette analyse a permis la réalisation d'une carte géomorphologique (Fig. 2). Sur la superficie étudiée, huit types de relief ont été distingués, et notamment: dû à la dénudation, dû à l'érosion fluviatile et à la dénudation, glaciaire, thermokarstique, fluvio-glaciaire, nival et cryogène, marin, ainsi que glaciers et formes constituant les névés. On y a également indiqué lacs gélés, eaux des fjords, courants de suspension et sites où apparaît une faune fossile. 
Tableau 1

Traits caractéristiques des unités photomorphiques

\begin{tabular}{|c|c|c|c|}
\hline $\begin{array}{c}\mathrm{N}^{\circ} \\
\text { de l'unité }\end{array}$ & Phototon & Structure & Texture \\
\hline 1 & blanc & amorphe & - \\
\hline 2 & blanc avec taches gris clair & tachetée & chaotique \\
\hline 3 & blanc avec taches gris foncé & tachetée & chaotique \\
\hline 4 & gris clair & amorphe & - \\
\hline 5 & gris clair à bandes blanches & à bandes, & à tresses \\
\hline 6 & gris clair à bandes grises & à bandes & parallèle-concentrique \\
\hline 7 & gris clair rayé de gris & à fines rayures & parallèle, concentrique \\
\hline 8 & gris clair rayé de gris foncé & rayée & parallèle \\
\hline 9 & gris & amorphe & - \\
\hline 10 & gris à bandes blanches & à larges bandes & dendritique \\
\hline 11 & gris à bandes blanches & à larges bandes & à grilles \\
\hline 12 & $\begin{array}{l}\text { gris avec bandes blanches isolées et } \\
\text { taches gris foncé }\end{array}$ & à larges bandes & parallèle \\
\hline 13 & gris à bandes blanches & à bandes & parallèle \\
\hline 14 & gris tacheté de blanc & à petites taches & chaotique \\
\hline 15 & gris rayé de blanc & rayée & à rayons \\
\hline 16 & gris rayé de gris clair & rayée & à rayons \\
\hline 17 & gris à bandes gris clair & à bandes & à tresses \\
\hline 18 & gris à bandes gris clair & à bandes & en éventail \\
\hline 19 & $\begin{array}{l}\text { gris à bandes et avec petites } \\
\text { taches gris foncé }\end{array}$ & à bandes et à taches & poreuse \\
\hline 20 & gris foncé & amorphe & - \\
\hline 21 & gris foncé à bandes blanches & à bandes fines & orientée. allongée \\
\hline 22 & gris foncé à bandes grises & à larges bandes & parallèle \\
\hline 23 & gris foncé tacheté de gris & à petites taches & poreuse \\
\hline
\end{tabular}

La comparaison des deux cartes (carte d'unités photomorphiques et carte géomorphologique) a démontré une concordance essez importante de limites des unités photomorphiques et des types de relief.

Les superficies aux phototons blancs $(1,2,3)^{1}$, à la structure amorphe ou tachetée représentent des glaciers avec zone de neige sec (27) et fortement irriguée $(29)^{2}$ ainsi que des crêtes aux versants altérés (3).

Les unités au fond gris clair $(4,5,6)$, à la structure amorphe et à bandes étroites, à la texture à tresses, correspondent au type du relief fluvio-glaciaire et, partiellement, marin. Ce sont des lits de rivières à plusieurs courants (12) et des cônes alluviaux (16) de même que deltas (13), plages (24) et courants de suspension (37).

1 Cf. les explications au tableau 1.

${ }^{2}$ Cf. la légende de la carte géomorphologique (fig. 2). 

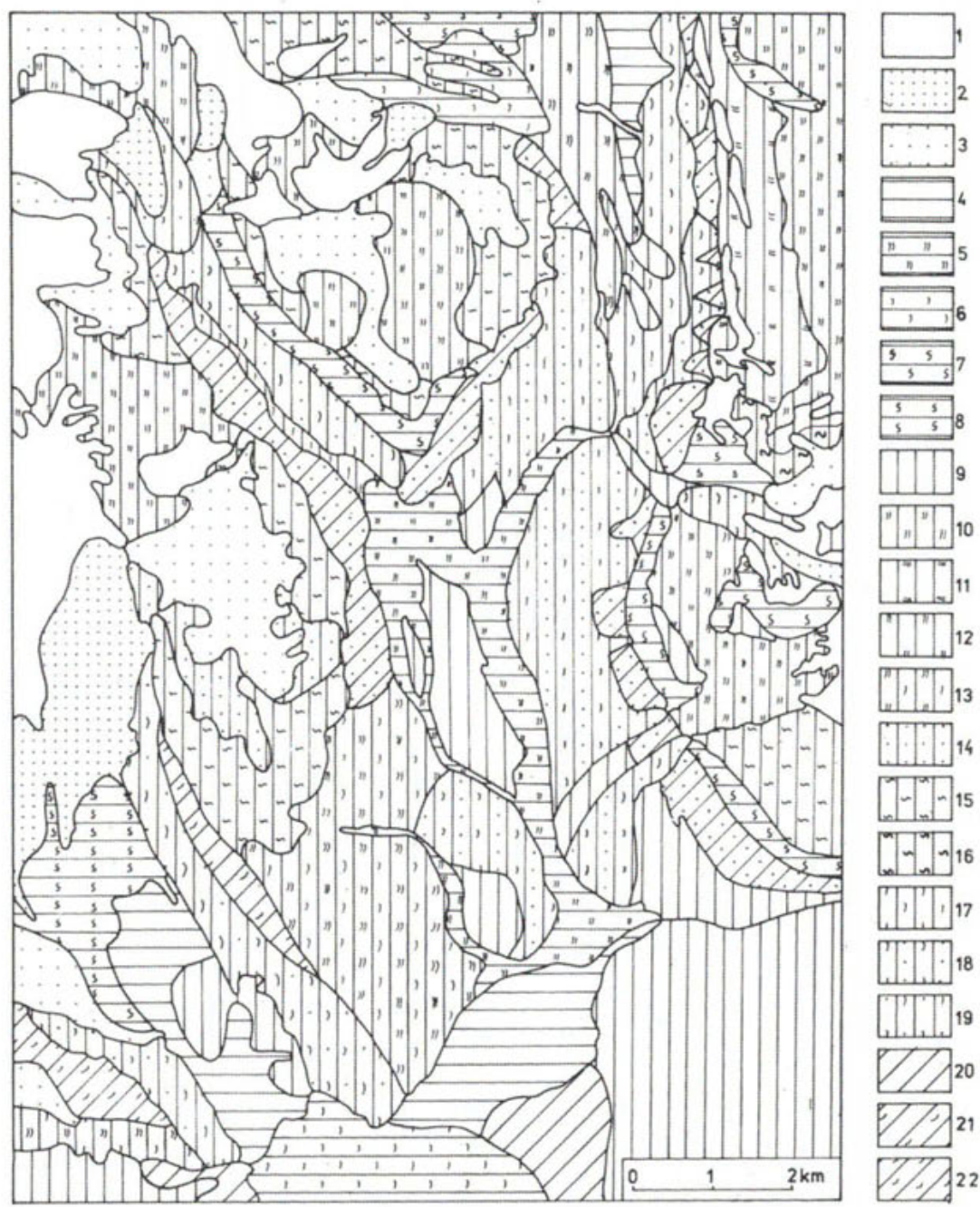

Fig. 1 Unités photomorphiques de la région de Berzeliusdalen (Spitsberg occidental). Pour les explications, cf. le tableau 1.

Les superficies au phototon gris clair $(7,8)$ et à la structure rayée, disposées parallèlement (texture régulière), représentent les ravins d'origine corrasivenivale (3) formés dans des grès tertiaires.

Les unités photomorphiques dont le fond est le phototon gris sont les plus nombreuses dans le terrain étudié, et les plus différenciées au point de vue de leur structure et la texture de l'image photographique, ainsi qu'au point du vue des formes de relief qui y ont été attestées.

Les superficies aux phototons gris, à la structure amorphe ou avec des bandes grises isolées $(9,13)$ représentent des formes d'origine marine c'est-à-dire des terrasses marines avec des levées de plage (26) et des escarpements (23). 


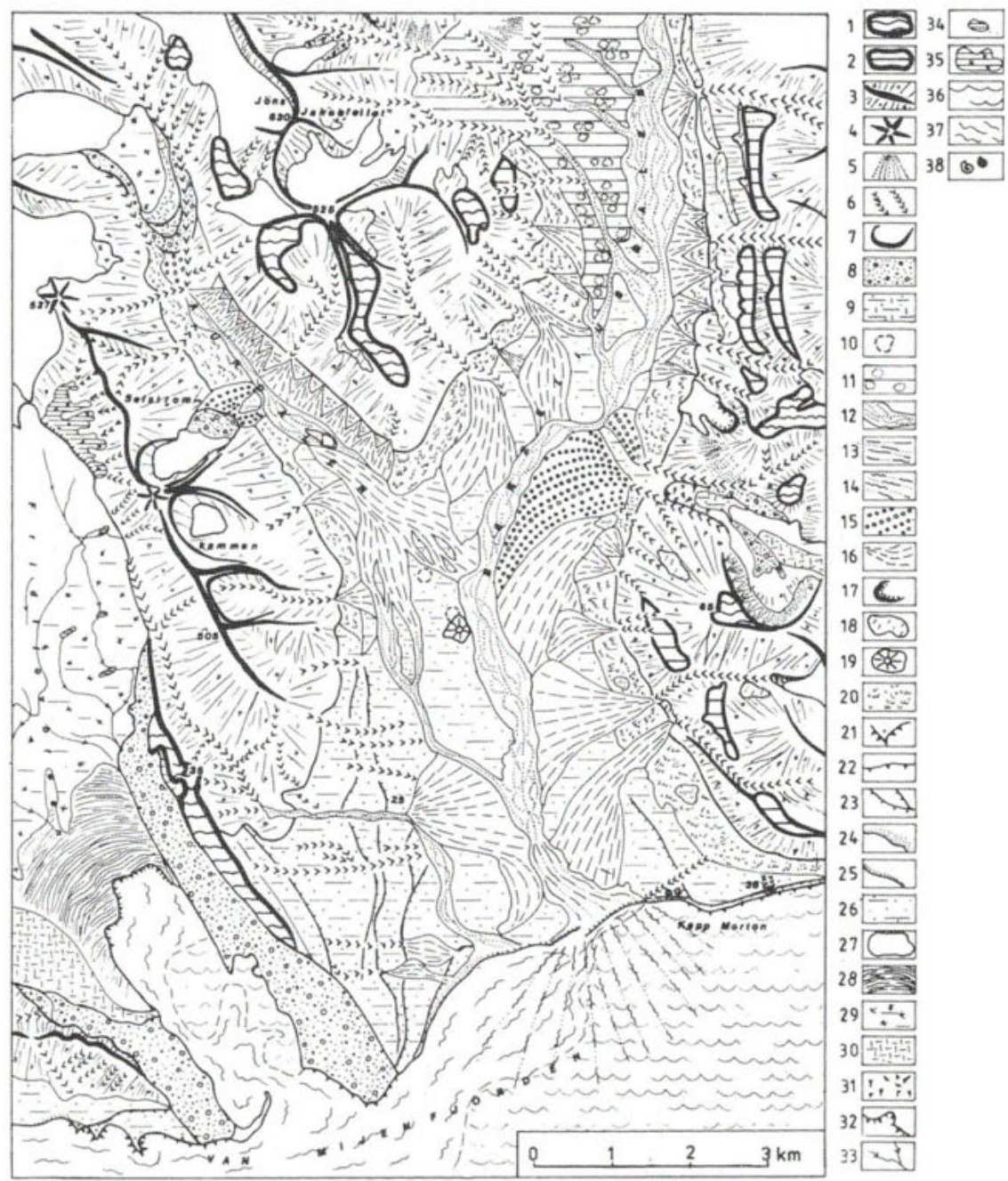

Fig. 2. Carte géomorphologique de la région de Berzeliusdalen (Spitsberg occidental)

FORMES DE DENUDATION. 1 - fragments d'anciennes surfaces, 2 - terrasses structurales, 3 - crêtes avec des versants aux surfaces altérées, 4 - sommets, 5 - cônes d'éboulis. FORMES DUES A L'EROSION FLUVIALE ET A LA DENUDATION. 6 - vallées en V. FORMES GLACIAIRES. 7 - cirques glaciaires, 8 - moraines frontales, centrales et latérales avec noyau glaciaire, 9 - moraine de fond sur glaces inactives. FORMES THERMOKARSTIQUES. 10 - cicatrices de pingo. FORMES FLUVIO-GLACLAIRES. 11 - fragments anciens des fonds de vallées avec des structures cryogènes, 12 - lits de rivières à plusieurs courants. 13 - delta, 14 - avant-delta, 15 - cônes fluvio-glaciaires, 16 - cônes alluviaux. FORMES NIVALES ET CRYOGENES. 17 - cirques nivaux, 18 - couloirs de neige plus importants, 19 - pingo, 20 - surfaces de solifluxion. FORMES MARINES. 21 - éperon d'abrasion, 22 - front de falaise, 23 -escarpements de terrasses marines, 24 - plages, 25 - flèches littorales, 26 - terrasses marines avec, par endroits, des levées de plage. NEVES ET GLACIERS. 27 - champs de neige surmontant les glaciers, 28 - zone des glaces avec nombreuses crevasses, 29 - champs de neige fortement irrigués surmontant les glaciers, 30 - glace pure des glaciers, 31 - glacier couvert de débris, 32 - front du glacier, 33 - ruisseaux supraglaciaires. INDICATIONS COMPLEMENTAIRES. 35 - lac gélé, 36 - eaux de fjords, 37 - courants de suspension, 38- sites 
L'unité 10 se dessine avec netteté sur la photo aérienne et se caractérise par le phototon blanc avec des bandes grises et larges lui conférant la texture dendritique. Elle correspond aux cirques glaciaires (1) et aux crêtes avec des versants aux surfaces altérées (3), coupées par des ravins corrasifs-nivaux et des vallées en $V(6)$.

L'unité photomorphique suivante (11) ne se distingue de la précédente que par la texture à grilles - résultat d'un croisement de vallées en $V(6)$ avec terrasses structurales (2) et des couloirs de neige (18).

Les unités photomorphiques 14-16 aux phototons gris, à la structure finement tachetée ou rayée et à la texture à rayons correspondent aux formes de dénudation. Les fragments supérieurs des versants aux surfaces altérées au-dessous de la ligne des crêtes (3) forment des superficies à la structure finement tachetée (unité 14). Aux fragments inférieurs des versants correspond l'unité 15 à la structure rayée dont l'image photographique reflète la présence d'un grand nombre de vallées en $\mathrm{V}$ (6). Aux formes d'origine fluvio-glaciaire correspondent les unités 12,17 et 18 au phototon gris, à la structure à bandes et à la texture différenciée, depuis la texture parallèle, en passant par la texture à tresses jusqu'à la texture en éventail.

La superficie 12 est formée de fragments anciens des fonds de vallées avec des structures cryogènes (11). L'unité 17 embrasse des lits de rivières à plusieurs courants et à la texture à tresses (12). L'unité 18 est la plus diversifiée quant aux formes qui y sont représentées. On y a reconnu des terrasses marines (23), des cônes alluviaux (16) et des cônes fluvio-glaciaires (15).

Les formes glaciaires, et notamment des moraines frontales, centrales et latérales avec noyau glaciaire (8), et des moraines de fond sur glaces inactives constituent l'unité 19 qui se caractérise par le phototon gris, la structure tachetée et rayée, et la texture poreuse.

Le dernier groupe d'unités photomorphiques, à savoir 20-23, se distingue par le phototon gris foncé, et la structure tachetée et à bandes. Les superficies isolées à la texture amorphe représentent les eaux des fjords (36), et les courants de suspension près de la côte (37). La texture parallèle caractérise d'anciennes surfaces (1) tandis que la texture poreuse - les formes nivales et les surfaces de solifluxion (20).

L'analyse du tableau 2 démontre qu'à l'origine du relief étudié se trouvent principalement des formes de dénudation ainsi que des formes fluvio-glaciaires et marines dont la part est importante.

Les unités photomorphiques aux phototons blanc, gris clair et gris foncé sont homogènes à un degré important avec les formes du relief. Par contre, le groupe d'unités aux phototons gris est plus diversifié, la plupart correspondant à deux types de relief.

L'analyse par photointerprétation de l'environnement naturel aux conditions naturelles intactes confirme la correspondance des limites d'unités photomorphiques aux contours des types de relief. Ainsi, Iorsqu'un type de relief correspond à une unité photomorphique, une analyse géomorphologique plus détaillée se 

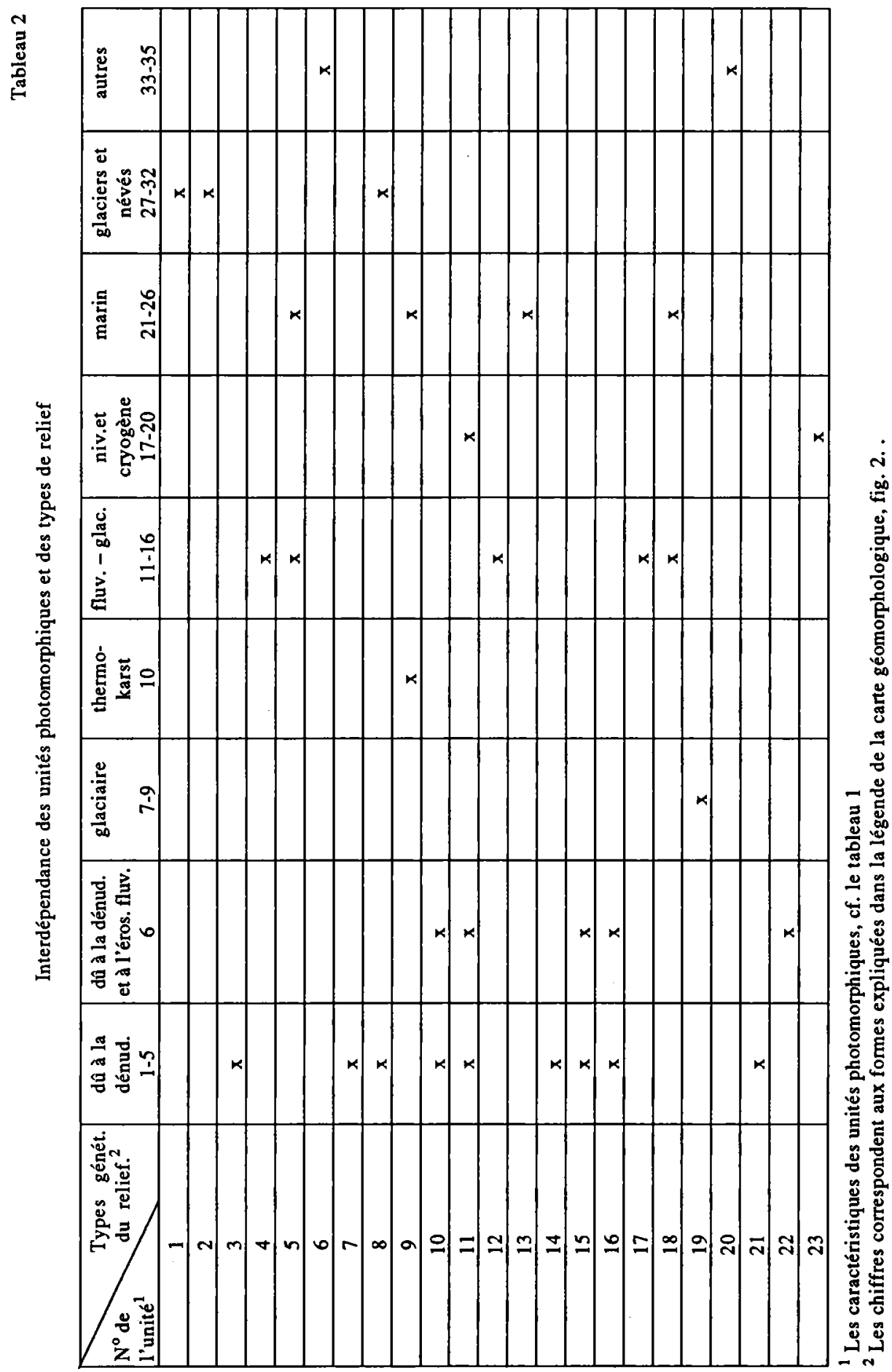
réduirait aux formes caractéristiques à un type donné. Donc, la crédibilité de l'interprétation du relief présenté sur les photos aériennes et, par là-même, de la carte géomorphologique exécutée à partir de cette photo serait plus grande.

Les terrains polaires sont difficiles à explorer, ainsi les unités photomorphiques qui apparaissent sur les matériaux de télédétection prennent une importance particulière. De plus, ces unités sont indispensables à des travaux cartographiques et permettent la reproduction exacte des contours des formes reconnues. 
